\title{
A Design of Wireless Communication Device based on Infrared Light
}

\author{
Bo ZHANG ${ }^{1, a}$, Jian-Ping XING ${ }^{1, b^{*}}$,Xiao-Ting SUN ${ }^{1, c}$ and Qing-Tao ZHAO ${ }^{1, d}$ \\ ${ }^{1}$ Shool of Information Science and Engineering, Shandong University, Jinan, China \\ azhangbo_sdu@163.com, bsduxingjianping@163.com, 'sdusxt@163.com, \\ dzhaoxiaohan12@163.com \\ * Corresponding author
}

Keywords: Infrared light, Wireless audio communication, Relay transmitting device.

Abstract. Infrared light communication is a kind of communication method that uses infrared ray to transmit information including voice, text, data, images and so on. In this paper, a kind of wireless communication device based on infrared light is designed, which uses the near-infrared band of 950nm to transmit voice information. The device is composed of infrared emitting device and infrared receiving device, and the relay transmitting device to make the signal be transmitted further. The infrared communication has the characteristics of large capacity, strong confidentiality, good immunity, simple structure, small size, and is widely used in toys and security.

\section{Introduction}

Infrared technology is the ancestor of wireless connection, which is an important part of advanced science and technology. The infrared transmission is a way to transmit voice and data with infrared light as a carrier by its spread in the air. Infrared communication is a kind of communication which is inexpensive, close quarters, wireless, low-power and high confidentiality. It's mainly applied to close quarters wireless data transmission and network access. Infrared interface communicates with directional infrared. It's widely applied in the small mobile devices because of its short wavelength and poor diffraction capability of obstacles, which is only suitable for short distance wireless communication occasions and point-to-point linear data transmission $[1,2,3]$. With the advances in science and technology, the rapid development of infrared technology brings big shock for the traditional voice transmission while bringing infinite hope and vitality for telecommunication industry. The application of infra-red technology makes voice wireless transmission possible and transmission more flexible, convenient and efficient. The infrared audio communication with faster transmission speed, low interference and high reliability, which is suitable for a variety of infinite close communication occasions, such as teachers' pronunciation teaching system, domestic bedroom, hospital, library, etc., has the official potential in the electronic products.

\section{System design and requirements}

The main content of this design is that obtaining the audio signal amplification which gets from audio input port without distortion using operational amplifier circuit, and input signal into firing circuit composed of infrared light-emitting channel to launch, and then receiving part obtains the received signal by infrared receiving ends, which will be amplified by audio power amplifier chip and output, and then finally get audio signal that is better quality. The expected target of this design is that the voice signal, whose transmission distance is at least $2 \mathrm{~m}$, and the frequency range is from $300 \mathrm{~Hz}$ to $3400 \mathrm{~Hz}$, is transmitted by directional transmission through the infrared emitting di-ode [4] and the infrared receiving module [5] is the transceiver. Relay forwarding node is added for the infrared communications to change the communication direction of $90^{\circ}$ and extend the communication distance of $2 \mathrm{~m}[6,7]$. There is no obvious distortion in the received sound signal. The system design requirements are shown in Fig 1. 


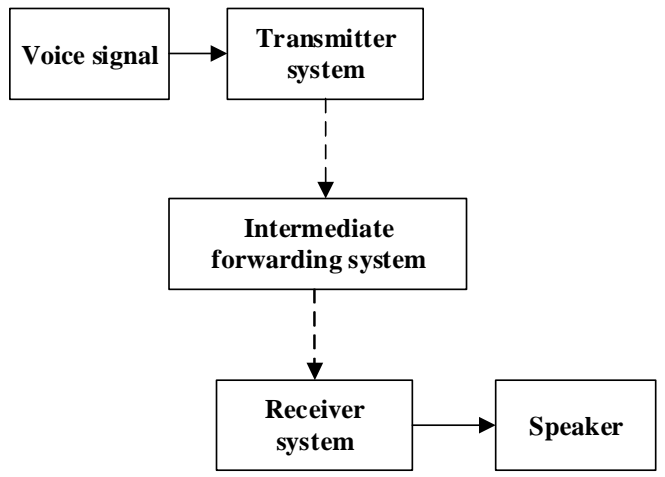

Fig 1. The design requirements of the infrared wireless audio communication.

\section{System scheme}

\section{Overall system design}

The infrared communication can be divided into the digital communication and the analog communication. Analog signal is used in this system. The analog signal input from the headphones will be transmitted through the stopping capacitor, and the audio power amplifier. The receiving end converts the optical signal which received by the infrared receiving channel into the electrical signal, and the signal is transmitted to the speaker trough the band pass filter and the audio power amplifier.

\section{Design of transmitter system}

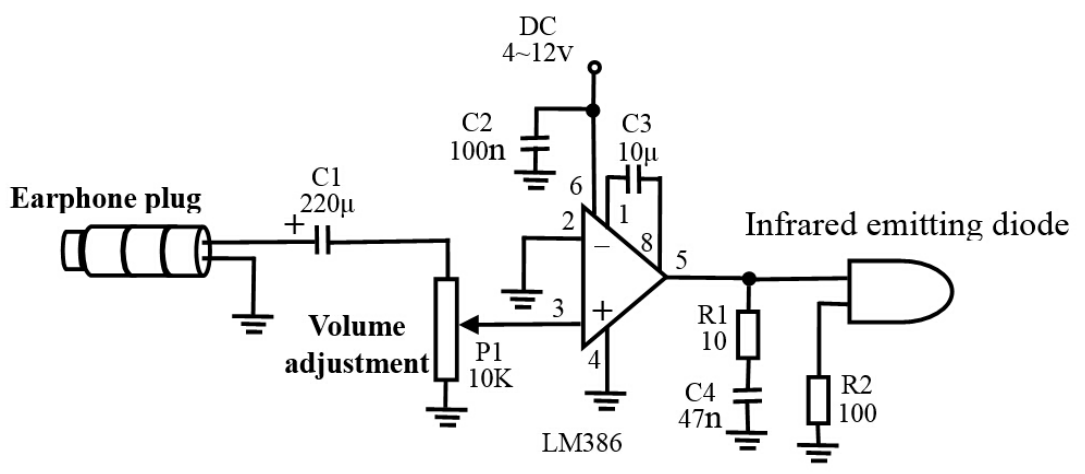

Fig 2. The circuit diagram of the transmitting system.

The circuit diagram of the transmitter system is shown in Fig 2. The electrical signal, which is received by the earphone plug, entered into the audio power amplifier through the stopping capacitor. LM386 [7] chip is used as the audio power amplifier. And this chip is a kind of audio integrated power amplifier, which has the advantages of low power consumption, adjustable internal chain gain, wide supply voltage range, and less outward element. The input signal is very weak, thus we can make the gain of 50-200 times, increase the transmission energy and en-large the receiving distance using the audio power amplifier LM386 before launching device. The amplified analog signal is emitted through an infrared emitting channel. In ad-dition, the infrared channel of the emission can be used in the spotlight to increase the transmission distance.

\section{Design of receiving system}

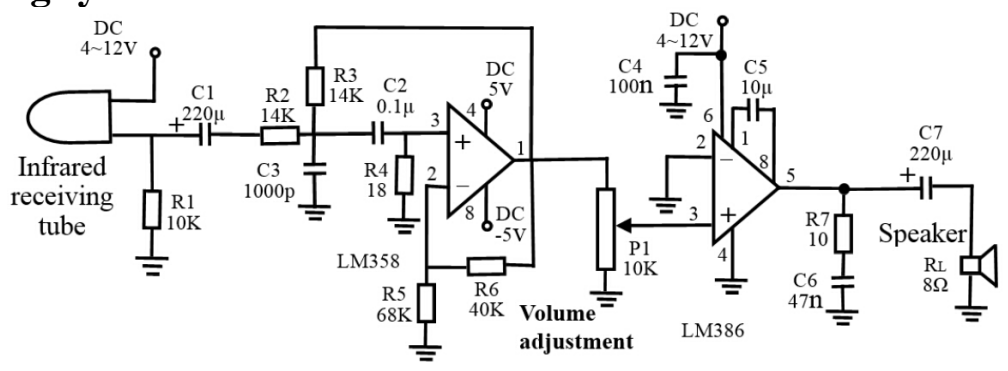

Fig 3. The circuit diagram of the receiving system. 
The circuit diagram of the receiving system [8] is shown in Fig 3. The optical signal, which received by the infrared receiving channel, is converted into the electrical signal through the band pass filter. The band pass filter LM358 is a dual operational amplifier [9], including two inde-pendent dual operational amplifier, which has the ad-vantages of high gain, and internal frequency compensa-tion. It can be used in a single power supply that the sup-ply voltage range is very wide and a dual power supply. Its applications include transducer amplifier, dc gain am-plifier module and all the other available the occasion of the use of single power supply operational amplifier. Therefore, the LM358 can be used as the band pass filter in the circuit, while adjusting the size of the capacitor re-sistor so that the frequency is set to $300-3400 \mathrm{~Hz}$, filtering out other frequency signals and noise such as Gauss white noise. The filtered signal is still weak and insufficient to drive the load horn to work. Therefore, the audio power amplifier LM386 is needed to amplify the filtered signal and then drive the horn to work normally. In addition, the infrared channel of the transmitter can be used in the spot-light to increase the transmission distance.

\section{Design of relay system}

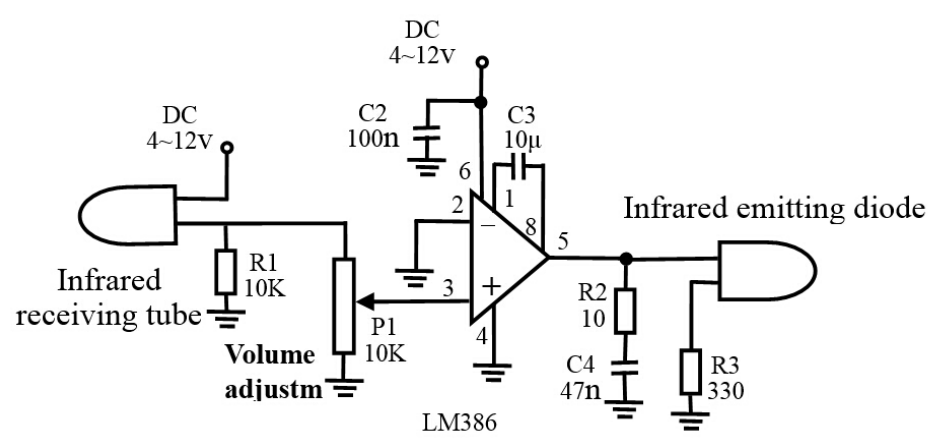

Fig 4. The circuit diagram of the intermediate forwarding system.

The circuit diagram of the intermediate forwarding system [10] is shown in Fig 4. Increasing the relay can extend the distance of the infrared communication, and the system design only needs two infrared emitting channel, two infrared-receiving channel and the audio power amplifier LM386. The infrared signal received by the infrared-receiving channel is amplified and transmitted through the infrared-emitting channel, so the relay system can play a role in enhancing the intensity of the infrared signal. Adjusting the size of the R3 can change the power of the relay. After testing, the size of R3 can be about $330 \Omega$ and the power consumption of relay is low.

\section{Test results of the design scheme}

When the voice signal from the transmitter input is the tone signal whose frequency is $800 \mathrm{~Hz}$, and the load re-sistance is $8 \Omega$, the effective value of the receiving output voltage is $0.442 \mathrm{~V}$. The state of the circuit is not changed, and the input signal at the transmitter is reduced to $0 \mathrm{~V}$. The value of the noise voltage at the output of the receiving device is $0.24 \mathrm{~V}$ with the low frequency millivoltmeter.

When the speech signal is inputted at the transmitter, the design of the circuit is achieved the basic requirements according to the parameters and the waveform of the speech signal from the test. The received sound has no obvious distortion, and the infrared transmission distance can be reached $8 \sim 10 \mathrm{~m}$, which is consistent with the basic requirements of the subject. The signal can be changed the direction of communication after 90 degrees, the transmission distance can be reached $10 \mathrm{~m}$ and the system can be reached the requirements of the design through the relay system.

\section{Conclusion}

The infrared emitting and receiving module are used as the transmitting-receiving device for the directional transmission of the analog speech signal based on the infrared light. The analog speech 
signal, whose frequency range is $300 \sim 3400 \mathrm{~Hz}$ and transmission distance can be reached $10 \mathrm{~m}$, is transmitted by audio jack line input or a low frequency signal source. In order to make the voice more clear, The noise, which is generated by the heat of the each component in the circuit and other interference signal (such as the infrared signal emitted by the objects, the electromag-netic radiation of other electrical appliances in the room, etc.), is reduced, and the magnification of the triode and the power amplifier is regulated, thus the minimum distortion of the sound signal is ensured. For longer transmission distance of signals, on the one hand the signal magnification of the receiver is improved as far as possible in the case of the smallest degree of distortion, on the other hand the transmitting power of the transmitter is regulated.

\section{Acknowledgments}

I would like to express my gratitude to all those who helped me during the writing of this thesis. My deepest gratitude goes first and foremost to Professor Jianping Xing, my supervisor, for his constant encouragement and guidance.

\section{References}

[1] Kahn, Joseph M., and John R. Barry. "Wireless infrared communications." Proceedings of the IEEE 85.2 (1997): 265-298.

[2] Barry, John R. Wireless infrared communications. Vol. 280. Springer Science \& Business Media, 2012.

[3] F. R. Gfeller and U. H. Bapst, "Wireless in-house data communication via diffuse infrared radiation”, Proc. IEEE, vol. 67, no. 11, pp. 1474-1486, Nov., 1979.

[4] Brabec, Christoph J., et al. "A low-bandgap semiconducting polymer for photovoltaic devices and infrared emitting diodes." Advanced Functional Materials 12.10 (2002): 709-712.

[5] Li, M. "LM386 low voltage audio power amplifier." National Semiconductor Corporation (1999).

[6] Cheng, C. H. E. N. G., and H. O. N. G. Long. "Design and implementation of practical infrared communication device [J]." Electric Power Automation Equipment 9 (2009): 031.

[7] T. Komine, J. H. Lee, S. Haruyama and M. Nakagawa, “ Adaptive equalization system for visible light wireless communication utilizing multiple white LED lighting equipment", IEEE Trans. Wireless Commun., vol. 8, no. 6, pp. 2892-2900, June, 2009.

[8] Hongling, Zhang, et al. "Design of Light Wave Receiver and Its Application in Fuse System." SPOTS--EXEMPLIFIED BY CHENGDU UNIVERSITY OF INFORMATION TECHNOLOGY... HE PING 922.2 (2014): 83.

[9] Instruments, Texas. LM358 Datasheet. 2014-02-27.http://www.ti.com/lit/ds/symlink/lm158-n.pdf.

[10] Saritha, B., et al. "DETECTION OF HEART MURMURS USING PHONOCARDIOGRAPHIC SIGNALS." 